\title{
RESEARCH
}

\section{Metabolite analysis-aided diagnosis of papillary thyroid cancer}

\author{
Jian Chen 1,2,3, Qingyuan Hu3, Hongwei Hou33, Shuo Wang2, Yunfei Zhang4, Yanbo Luo3, Huan Chen³, Huimin Deng3, \\ Hongfu Zhu' ${ }^{3}$, Lirong Zhang ${ }^{5}$, Hansong Liu ${ }^{6}$, An Wang ${ }^{1}$ and Yong Liu ${ }^{1}$
}

IInstitute of Applied Technology, Hefei Institutes of Physical Science, Chinese Academy of Sciences, Hefei, China 2University of Science and Technology of China, Hefei, China

${ }^{3}$ Quality Safety and Framework Convention on Tobacco Control Research Team, China National Tobacco Quality Supervision \& Test Center, Zhengzhou, China

${ }^{4}$ Henan Cancer Hospital, Zhengzhou University, Zhengzhou, China

${ }^{5}$ School of Basic Medical Sciences, Zhengzhou University, Zhengzhou, China

${ }^{6}$ Zhengzhou Central Hospital Affiliated to Zhengzhou University, Zhengzhou, China

Correspondence should be addressed to H Hou or A Wang or Q Hu: qsfctc@163.com or wangan@aiofm.ac.cn or huqy1965@163.com

\begin{abstract}
Thyroid cancer is the most frequent endocrine tumor with a growing incidence worldwide. However, common diagnostic strategy for thyroid cancer classification is hardly to make a proper diagnosis in some cases. To assist classical approach, this study used metabolomics to screen and validate biomarkers from serum and urinary for papillary thyroid cancer (PTC). Overall, 124 untreated PTC, 76 untreated benign thyroid nodule (BTN), and 116 healthy control (HC) were collected in this study. Thirty-six differential metabolites were screened from non-targeted metabolomics with a discovery sample set in comparison with $\mathrm{HC}$ and BTN. Serum $\beta$-hydroxybutyrate (BHB), docosahexaenoic acid (DHA), 1-methyladenosine (1-MedA), pregnanediol-3-glucuronide (PdG), urinary nicotinic acid mononucleotide (NAM) and xanthosine (Xan) were validated to be significantly differential by targeted metabolomics in validation set. The logistic regression model incorporating six biomarkers had excellent discrimination from receiver-operating characteristics (ROC) analysis, with area under the receiver-operating characteristic curve (AUC) of 0.943 (95\% Cl 0.902 to 0.983 ) and 0.952 (95\% Cl 0.921 to 0.983 ) for female alone and female + male samples, respectively. The prediction accuracy and false-negative rate in the real setting of one PTC to ten suspicious nodules were 84.7 and $17.7 \%$ with the threshold at probablity of 0.5 . Results of a double-blind study for PTC and BTN had true positive value of $100 \%$ and true negative value of $91.7 \%$. To conclude, BHB, DHA, 1-MedA, PdG, NAM and Xan are suitable biomarkers for PTC, and logistic regression models with the six biomarkers can be potentially used as clinical diagnosis.
\end{abstract}
Key Words
- papillary thyroid carcinoma
- biomarker
- logistic regression model
- metabolomics
- double-blind

\section{Introduction}

Thyroid cancer is a common endocrine malignancy with an increasing incidence worldwide (La et al. 2015). In particular, papillary thyroid cancer (PTC) accounts for almost $90 \%$ of all thyroid tumors. Although the overall survival rate of thyroid cancer patients is high, it is worrying that the recurrence rate of 10 years is up to $30 \%$, and the survival rate of these high-risk patients is about $50 \%$. Ultrasound-guided fine-needle aspiration (FNA) biopsy, 
as the best imaging method to diagnose high-risk cancer lesions, depends heavily on the technical advantages and experience of the operator (Yao et al. 2011, Ravetto et al. 2000 ), which has a sensitivity of $93 \%$ and specificity less than $75 \%$. However, in $10-30 \%$ cases, a reliable diagnosis of malignant tumors could not be performed by cytological analysis of FNA samples, mainly because it is difficult to distinguish between benign and malignant follicular thyroid lesions (Wang et al. 2011). Although FNA cytological analysis is popular and useful, immunocytochemical and genetic tests still have some limitations (Lin et al. 2017, Wojtowicz et al. 2017, Coperchini et al. 2019). Furthermore, indolent thyroid cancer does not require FNA biopsy unless there is a lymph node metastasis, extrathyroidal invasion, or previous exposure to radiation or a family history of thyroid cancer, and the treatment of these over-diagnosed cancers may increase the risk of patient. To prevent patients from unnecessary injuries and improve diagnostic accuracy, a convenient, noninvasive and specific diagnosis method is urgently to be developed for thyroid cancer (Wojtowicz et al. 2017).

Measuring small-molecule metabolites is essentially a cornerstone of clinical chemistry and even clinical practice. Molecular markers, mainly found in blood/ serum, urine, other body fluids, or tissues, are expected to have a significant impact on cancer prognostication (Haugen et al. 2016, Pan et al. 2016, Wang et al. 2017a). Metabolomics is a relatively new field for detecting biochemical changes associated with genetic differences, environmental impacts, diseases or drug interferences (Nicholson \& Lindon 2008, Ceglarek et al. 2009, Dunn et al. 2011, Zafón Llopis et al. 2019), and may be useful for characterizing the pathogenicity of gene variants and their levels of aggressiveness (Veyrat-Durebex et al. 2019). Thyroid cancer showed obvious metabolic heterogeneity in tumor microenvironment, and there was metabolic coupling between tumor and stromal cells. The actual understanding of the metabolic phenotype of thyroid cancer cells will provide new prognostic biomarkers and therapeutic targets for aggressive tumor behavior (Wojakowska et al. 2015a). Metabolic biomarkers are hopefully to be incorporated into diagnostic and therapeutic practices to improve the outcomes of thyroid cancer, which may also be used for early detection or screening, differentiation of benign from malignant diseases, histologic identification, staging and response to treatment, diagnosis of recurrence and prognosis. These targets have generated considerable interest in identifying promising tumor markers (Bustamante et al. 1981, Wise \& Thompson 2010, Locasale 2013, Goodwin et al. 2014).
Metabolites related to lactic acid metabolism ( $\mathrm{Xu}$ et al. 2015), one carbon metabolism (Ryoo et al. 2016), fatty acids metabolism (Tian et al. 2015) and lipid metabolism (Wojakowska et al. 2015b) were found to be significantly altered in thyroid cancer. Despite promising progress in serum and urine metabolomics of thyroid cancer, there are still some shortcomings, such as lack of validation or limited validation biomarkers of thyroid cancer early diagnosis.

To fill this gap, our most urgent interest is to develop a diagnostic strategy-based metabolomics study to help early diagnosis of PTC. Thirty-six metabolites were screened by non-targeted metabolomics in discovery set, and six differential metabolites were proved to be proper biomarkers for PTC by quantitation with validation set. A new biomarker panel based on logistic regression model with ROC analysis and bootstrapping validation was applied to estimate the probability of PTC from non-cancer group (healthy control (HC) and benign thyroid nodule (BTN)). Moreover, a prospective, randomized double-blinded study of 31 untreated patients with thyroid nodules was conducted to validate the performance of the logistic regression model.

\section{Materials and methods}

\section{Clinical and pathologic characteristics of thyroid cancer}

Samples of Han Chinese volunteers, including 124 serum/ urine samples from untreated PTC, 76 serum/urine samples from untreated BTN, and 116 serum/urine samples from HC, were collected from the Tumor Hospital affiliated to Zhengzhou University and Zhengzhou Central Hospital affiliated to Zhengzhou University with ultrasound, FNA biopsy and histopathology. Tumor staging was performed according to the eighth edition of thyroid cancer tumor node metastasis staging by American Joint Committee (van Velsen et al. 2018). This study was approved by the Ethics Committee of School of Basic Medical Sciences, Zhengzhou University (approval number 18), and all participants received informed consent. HC were recruited and asked to undergo health checks to ensure everyone without cardiovascular, thyroid, liver, kidney or blood system dysfunction were excluded. The fasting serum sample was collected in the morning, and set at $4^{\circ} \mathrm{C}$ for $2 \mathrm{~h}$, and then centrifuged for $15 \min \left(3000 \mathrm{~g}, 4^{\circ} \mathrm{C}\right)$ (Dunn et al. 2011). Multiple aliquots were collected for each subject and stored at $-80^{\circ} \mathrm{C}$ until further analysis. Histopathologic classification was performed according to the World Health Organization (WHO) Classification (c) 2019 Society for Endocrinology Published by Bioscientifica Ltd. Printed in Great Britain 
of Tumours of Endocrine Organs $4^{\text {th }}$ Edition by two pathologists (Lloyd et al. 2017). The baseline characteristics of enrolled patients and HC were listed in Table 1. Each discovery set has 58 subjects, including 29 female PTC stage I/II patients, 29 non-cancer subjects (15 HC and 14 female BTN patients), and the average ages of PTC stage I/II patients and non-cancer subjects were 43.7 and 43.7 years old, respectively. Quality control (QC) samples were mixture of female PTC, BTN and HC samples with same volume before preparation. An independent validation set with 166 female samples (62 PTC stage I/II, 4 stage III/IV, 64 HC and 36 BTN) was used to discover and verify the potential biomarkers (Table 1 ). In addition, male PTC test set was consisted of 28 PTC stage I/II, 2 stage III/IV,
$37 \mathrm{HC}$ and 2 BTN. Moreover, a double-blinded set was consisted of 31 untreated patients with thyroid nodules.

\section{Development and validation of the untargeted metabolomics method}

\section{Apparatus}

All chromatography separations were conducted with the Thermo Fisher Scientific Ultimate 3000 UHPLC system (including HPG-3400 SD pump, WPS-3000 SL auto-sampler and TCC-3000 SD column oven). The MS detection was performed on a Bruker microOTOF-Q II (Bruker Daltonics Corporation, Karlsruhe, Germany) using an electronic spray ion source.

Table 1 Baseline clinical and pathological features of enrolled patients and controls.

\begin{tabular}{|c|c|c|c|c|}
\hline & $\mathrm{HC}$ & BTN & Stage I & $P$ value (ANOVA) \\
\hline Cases & 15 & 14 & 29 & \\
\hline Age (average, range) & $43.7,24-57$ & $43.8,24-54$ & $43.7,23-55$ & $P=0.864$ \\
\hline \multicolumn{5}{|l|}{ Tumor stage } \\
\hline $\mathrm{T} 1 / 2$ & & & 29 & \\
\hline $\mathrm{T} 3 / 4$ & & & 0 & \\
\hline \multicolumn{5}{|l|}{ Lymph node metastases } \\
\hline NO & & & 26 & \\
\hline N1 & & & 3 & \\
\hline \multicolumn{5}{|l|}{ Distant metastases } \\
\hline MO & & & 29 & \\
\hline M1 & & & 0 & \\
\hline \multicolumn{5}{|c|}{ (2) Baseline clinical features of enrolled patients and controls of external validation set (female) } \\
\hline & $\mathrm{HC}$ & BTN & Stage I/II & Stage III/IV \\
\hline Cases & 64 & 36 & 62 & 4 \\
\hline Age (average, range) & $41.8,26-68$ & $46.8,24-58$ & $43.8,20-74$ & $66.5,63-69$ \\
\hline \multicolumn{5}{|l|}{ Tumor stage } \\
\hline $\mathrm{T} 1 / 2$ & & & 53 & 0 \\
\hline $\mathrm{T} 3 / 4$ & & & 9 & 4 \\
\hline \multicolumn{5}{|l|}{ Lymph node metastases } \\
\hline NO & & & 45 & 1 \\
\hline N1 & & & 17 & 3 \\
\hline \multicolumn{5}{|l|}{ Distant metastases } \\
\hline MO & & & 60 & 1 \\
\hline 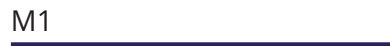 & & & 2 & 3 \\
\hline \multicolumn{5}{|c|}{ (3) Baseline clinical features of enrolled patients and controls of external test set (male) } \\
\hline & $\mathrm{HC}$ & BTN & Stage I/II & Stage III/IV \\
\hline Cases & 37 & 2 & 28 & 2 \\
\hline Age (average, range) & $34.2,23-62$ & $41.0,31-51$ & $42.4,21-66$ & $61.5,59-64$ \\
\hline \multicolumn{5}{|l|}{ Tumor stage } \\
\hline $\mathrm{T} 1 / 2$ & & & 26 & 0 \\
\hline $\mathrm{T} 3 / 4$ & & & 2 & 2 \\
\hline \multicolumn{5}{|l|}{ Lymph node metastases } \\
\hline NO & & & 17 & 0 \\
\hline N1 & & & 11 & 2 \\
\hline \multicolumn{5}{|l|}{ Distant metastases } \\
\hline MO & & & 27 & 0 \\
\hline M1 & & & 1 & 2 \\
\hline
\end{tabular}




\section{Chromatographic conditions}

An ACQUITY UPLC ${ }^{\circledR}$ BEH column $(2.1 \times 100 \mathrm{~mm}, 1.7 \mu \mathrm{m})$ (Waters Corporation) was used. RP-UHPLC mobile phases were consisted of A ( $0.1 \%$ formic acid) and B (acetonitrile with $0.1 \%$ formic acid). HILIC-UHPLC mobile phases were consisted of A $(0.1 \%$ formic acid and $10 \mathrm{mM}$ ammonium acetate in water) and B (0.1\% formic acid and $10 \mathrm{mM}$ ammonium acetate in acetonitrile). Injection volume and column temperature were $5 \mu \mathrm{L}$ and $50^{\circ} \mathrm{C}$, respectively. Gradient elution programs of RP-HUPLC and HILIC-UHPLC in positive and negative mode were displayed in Supplementary Table 1 (see section on supplementary data given at the end of this article).

\section{ESI-QTOF/MS conditions}

The MS detection was obtained on a Bruker microQTOF-Q II (Bruker Daltonics Corporation, Karlsruhe, Germany) equipped with an electronic spray ion source operating in negative ion mode. Argon was used as collision gas. The parameters were set as follows: Temperature of source, $180^{\circ} \mathrm{C}$; spray voltage, $3500 \mathrm{~V}$; flow rate of dry gas, $6 \mathrm{~L} / \mathrm{min}$; nebulizer gas, 1.5 bar; transmission time, $60 \mu \mathrm{s}$; collision energy, $7 \mathrm{eV}$; mass range, 50-1000 Da. We used sodium formate to correct the mass accuracy.

\section{ESI+QTOF/MS conditions}

The MS detection was obtained on a Bruker microQTOF-Q II (Bruker Daltonics Corporation) equipped with an electronic spray ion source operating in positive ion mode. Argon was used as collision gas. The settings are as follows: Temperature of source, $180^{\circ} \mathrm{C}$; spray voltage, $3500 \mathrm{~V}$; flow rate of dry gas, $4 \mathrm{~L} / \mathrm{min}$; atomizer gas, $0.04 \mathrm{MPa}$; transmission time, $100 \mu$ s; collision energy, $7 \mathrm{eV}$; mass range, 50-1000 Da. Mass accuracy was also corrected by sodium formate.

\section{GC-MS conditions}

GC-MS analysis was performed with Shimadzu GC-MS QP2010 coupled with a DB-5MS capillary column $(60 \mathrm{~m} \times 250 \mu \mathrm{m}$ i.d., $0.25 \mu \mathrm{m}$ film thickness; Agilent Technologies). Solvent delay was set for $3.5 \mathrm{~min}$. The measurements were conducted with electron impact ionization $(70 \mathrm{eV})$ under the full scan mode $(\mathrm{m} / \mathrm{z} 45-600)$. The oven temperature was initially held at $100^{\circ} \mathrm{C}$ for $4 \mathrm{~min}$. Then raised the temperature with a gradient of $4^{\circ} \mathrm{C} / \mathrm{min}$ to $320^{\circ} \mathrm{C}$ and held for $8.0 \mathrm{~min}$. The injection temperature, the interface temperature and the ion source temperature were set to $230^{\circ} \mathrm{C}, 280^{\circ} \mathrm{C}$ and $200^{\circ} \mathrm{C}$, respectively. The flow through the column was constant with $2.2 \mathrm{~mL} \mathrm{He} / \mathrm{min}$.
The compound libraries, Smartdatabase (OA_TMS_DB5_ 67min_V3.lib) and National Institute of Standards and Technology (NIST) and reference compounds available, were commercially available to identify metabolites.

\section{Data preprocessing}

Raw MS data for each sample was uploaded to XCMS online, and then extracted and overlaid variables (the integral results of peaks) for all the samples according to retention time or accurate mass data. In order to process the data as a whole, multivariate analysis was used to observe the differences between groups. Orthogonal projections to latent structure-discriminant analysis (OPLS-DA) models by SIMCA software were outlined to overview the differences in metabolic profiling between the PTC and non-cancer. The results were shown in Fig. 1, where $\mathrm{X}$ is the numerical value of the targeted steroids, and $\mathrm{Y}$ is the class assignment. It was revealed that the PTC patients were obviously separated from the non-cancer. The models were validated using a permutation test with 200 cycles. $P<0.05$ (CV-ANOVA) indicated that the OPLS-DA models were not over fitting.

The sequential processing of the peak table used the raw data output (including RT and characteristic ions) after the operation of the quantization table and the peak area was normalized to the total peak area. Principal component analysis (PCA) and OPLS-DA models were performed using SIMCA 14.1 (Umetrics, Sweden). Nonparametric tests (Mann-Whitney $U$ test) were performed with SPSS 22.0 software to screen for the metabolites of $P<0.05$. Changes in differential metabolite levels were observed in MultiExperiment Viewer (http:// www.tm4.org/mev.html). The altered pathways were identified by the Human Metabolome Database (HMDB), MetaboAnalyst 3.0 (http://www.metaboanalyst.ca), and KEGG PATHWAY Database

\section{Targeted analyses: identification and quantitation of marker compounds and creatinine}

From the standpoint of application, an additional validation sample was also acquired. The validated differential metabolites were further screened for the discovery of potential biomarkers for PTC diagnosis. To confirm whether potential biomarkers screen for PTC diagnosis, six validated differential metabolites were quantified in female external validation set subjects, both alone and combined with male external test set subjects; (c) 2019 Society for Endocrinology Published by Bioscientifica Ltd. Printed in Great Britain 

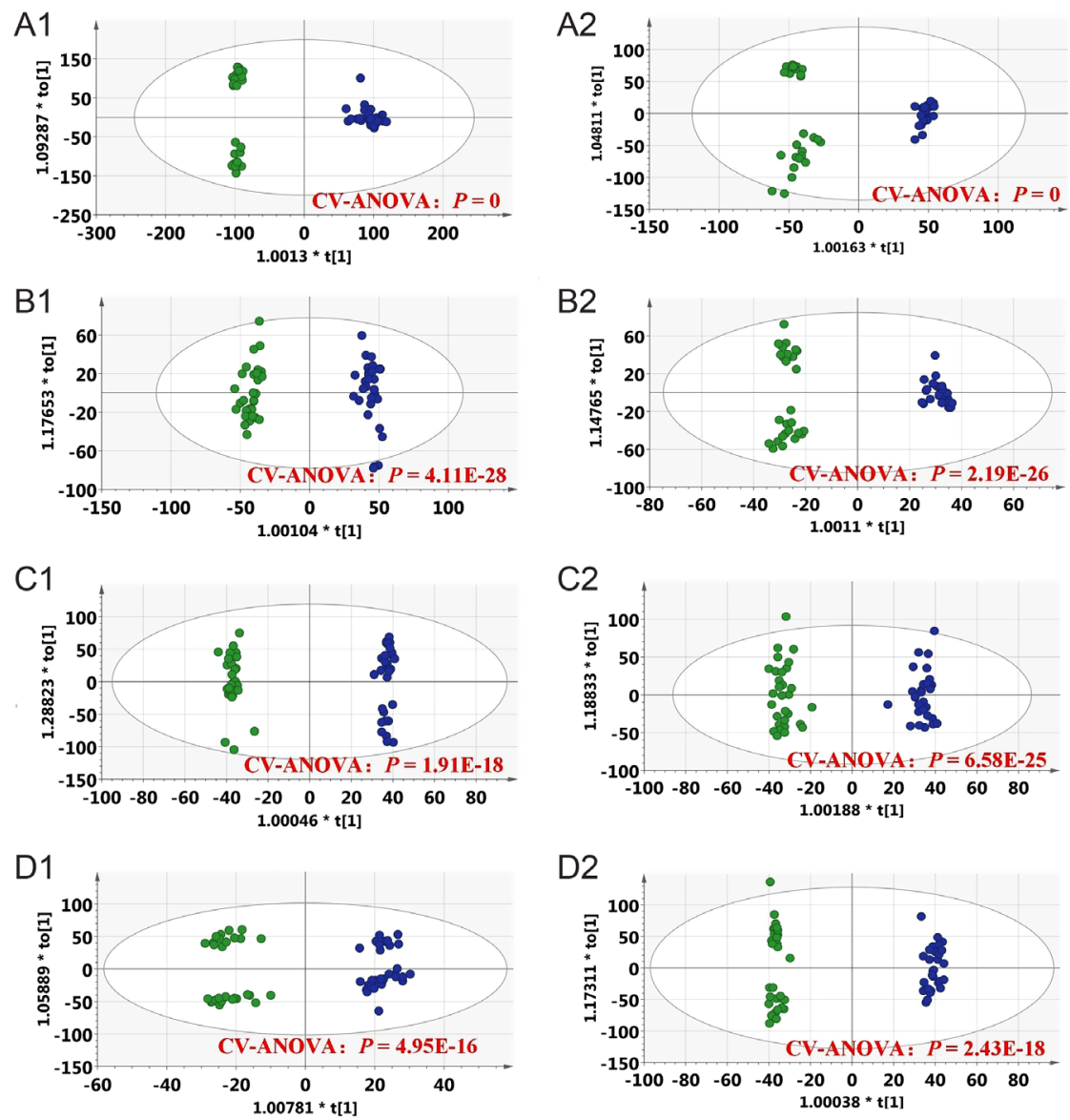

\begin{abstract}
Figure 1
Orthogonal projections to latent structurediscriminant analysis (OPLS-DA) models (green for control and red for PTC): (A1) serum HPLC-ESI(+)QTOF-MS, (A2) urinary HPLC-ESI(+)-QTOF-MS, (B1) serum HPLC-ESI(-)-QTOF-MS, (B2) urinary HPLC-ESI(-)-QTOF-MS, (C1) serum HILIC-ESI(+)QTOF-MS. (C2) urinary HILIC-ESI(+)-QTOF-MS, (D1) serum HILIC-ESI(-)-QTOF-MS, (D2) urinary HILIC-ESI(-)-QTOF-MS, (E1) serum GC-MS. A full colour version of this figure is available at https:// doi.org/10.1530/ERC-19-0344.
\end{abstract}

the significant changes among groups were verified by SPSS 22.0 software. Representative ESI product ion mass spectra of the precursor ions of markers and total ion chromatogram (TIC) were shown in Supplementary Fig. 1. Detection of creatinine in urine was pretreated and detected according to previous method (Jones et al. 2018).

\section{Risk prediction model}

Logistic regression models were widely used in medicine to predict patient outcome (prognosis) and construct diagnostic tests (diagnosis). Multivariable logistic regression models built with Matlab were constructed to estimate the probability. The performance of the multivariable model was quantified by assessing its discrimination and calibration. Model calibration was evaluated using Hosmer-Lemeshow goodness-of-fit test, together with the deviance and residual test. The overall model performance was evaluated using the Brier score. Bootstrap resampling (1000 cycles) was used for internal validation of the risk model, which was created in each cycle and corrected for optimism to evaluate how much the model performance decrease when applied in new data sets. ROC curve is a plot of all the (false-positive rate (FPR), true-positive rate (TPR)) pairs by varying the decision threshold over the whole range of the bootstrap sampling distributions, providing a more comprehensive evaluation on the model. Sensitivity and specificity at a probability threshold of 0.5 were calculated through ROC analysis.

As permutation analysis might overestimate the accuracy, we repeated 1000 cycles to extract one PTC 
to ten suspicious nodules as the prediction set, and calculated the predicted accuracy and false-negative rate with logistic regression model built on the leaving samples. Besides that, a prospective, randomized doubleblinded study of 31 untreated patients with thyroid nodules was conducted to assess the performance of the logistic regression model. The results of the laboratory metabolic markers will be unknown to the clinicians who will make the diagnosis, while the results of the clinical diagnosis will not be known to the laboratory technician who will determine these metabolic markers.

\section{Results}

\section{Differentiation between PTC patients and non-cancer group based on metabolomic profiles}

OPLS-DA models were outlined to overview the differences in metabolism profile between PTC and non-cancer $(\mathrm{HC}+\mathrm{BTN})$ (Fig. 1). The results revealed that PTCs were obviously separated from non-cancer. $P<0.05$ (CV-ANOVA) indicates that the OPLS-DA models were not overfitting. The samples of non-cancer group and PTC group were successfully separated in Fig. 1, demonstrating that specific metabolites are different between two groups. Based on the above models, differential metabolites passing VIP threshold (VIP > 1) and Welch's t-test $(P<0.05)$ were selected. The differential metabolites were identified by searching databases or compared with the spectra of known standard compounds. Thirty-six potential differential metabolites identified by high-throughput screening were included in serum or urine (Fig. 2).

Pooled QC samples were used in the study to make sure that there is no or minimal loss of metabolic information. The analysis sequence consists of QC samples and subject samples. At the beginning of each analysis batch, six QC samples were injected, and then QC samples were analyzed every eighth times. In order to process the data as a whole, multivariate analysis was used to observe the differences between groups. Score scatter plots of PCA based on 36 differential metabolites (RSD $<20 \%$ ) revealed that the QC samples were closely assembled in the center (Fig. 3). The results showed that there was no drift during metabolomics profiling, and the stability and reproducibility were acceptable.

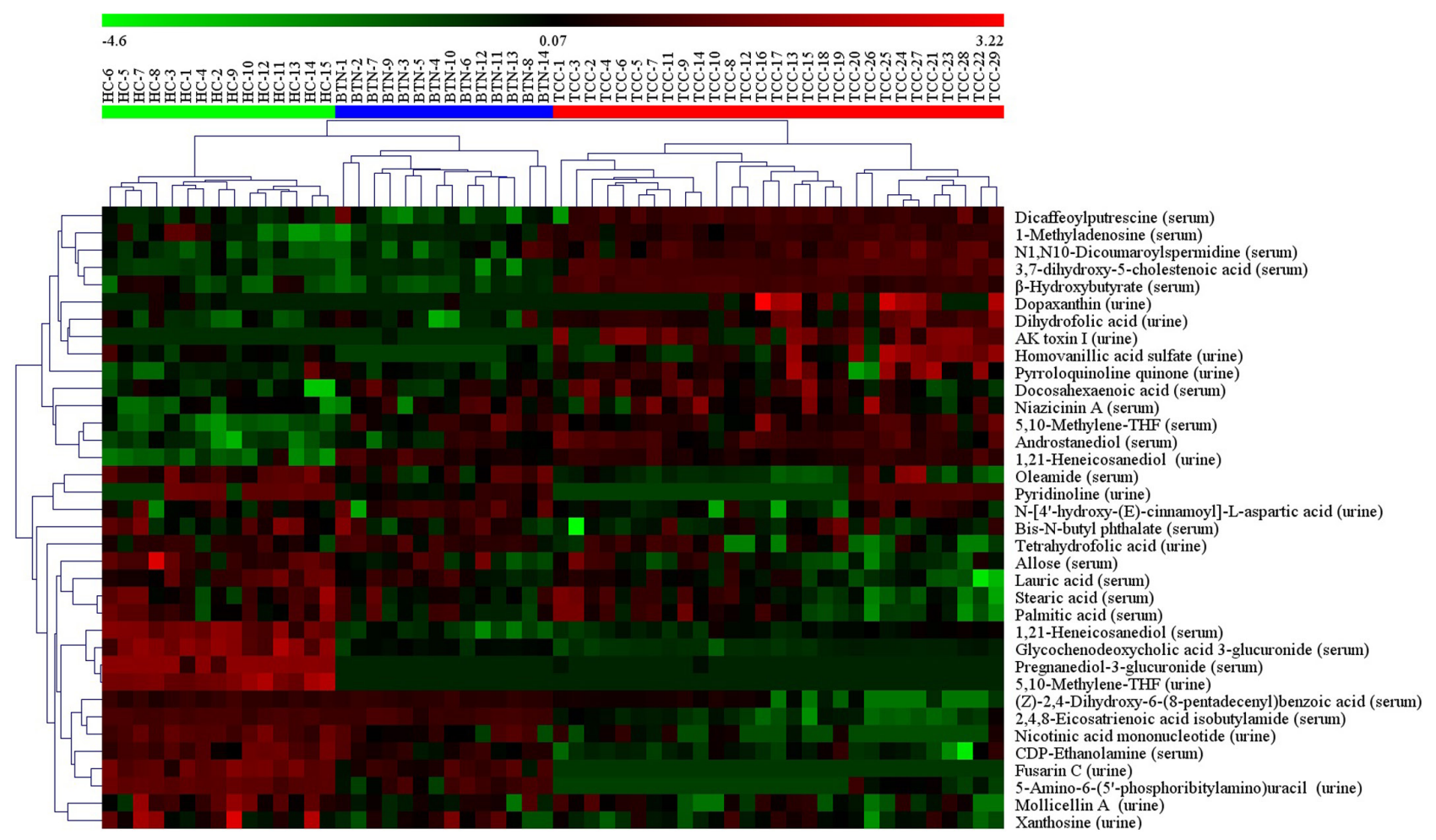

Figure 2

Heat map of identified differential metabolites screened by untargeted metabolomics in discovery set samples. A full colour version of this figure is available at https://doi.org/10.1530/ERC-19-0344. 


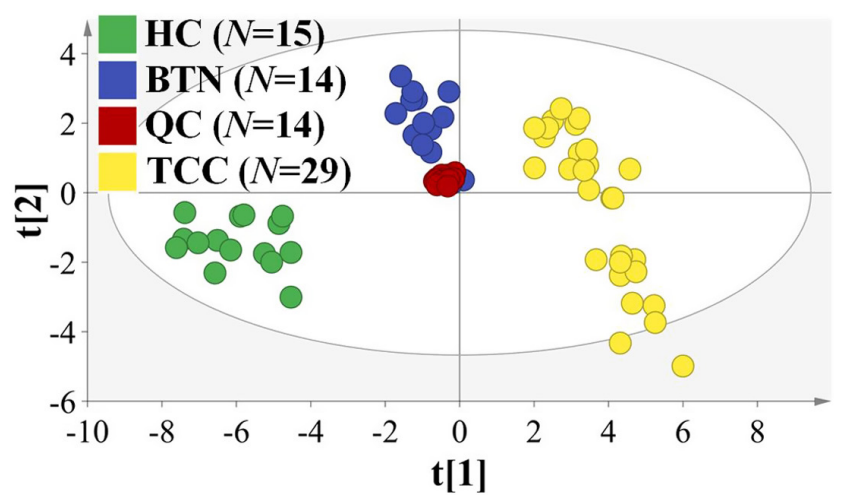

Figure 3

Score scatter plots of principal component analysis (PCA) based on 36 differential metabolites: QC samples were closely assembled in the center. A full colour version of this figure is available at https://doi. org/10.1530/ERC-19-0344.

\section{Dynamics of metabolite alterations in PTC}

Six differential metabolites were selected as candidate markers for further testing as they represented different metabolic pathways associated with cancer and had more significant differences. Serum $\beta$-hydroxybutyrate (BHB), docosahexaenoic acid (DHA) and 1-methyladenosine (1-MedA) were significantly increased in PTC. On the contrary, serum pregnanediol-3-glucuronide (PDG), urinary nicotinic acid mononucleotide (NAM) and xanthosine (Xan) significantly decreased (Fig. 2), which were rarely used as biomarkers for PTC, and they were mainly involved in fatty acid biosynthesis (Rodrigues et al. 2017), purine metabolism (Stoychev et al. 2010, Ishiwata et al. 1995), and nicotinate and nicotinamide metabolism (Wang et al. $2017 b$ ). As the differential metabolites were found mainly based on the difference of peak area of the compounds from a small sample experimental result, it is necessary to carry out the verification experiment by quantitative LC-MS/MS method (Supplementary Fig. 1) and correct with creatinine (Cre) in validation set (Table 1) (Levey et al. 1988). Compared to previously untargeted results (Fig. 2), these metabolites were proved to be potential biomarkers for PTC (Fig. 4). Serum BHB, DHA and 1-MedA were upregulated in PTC compared with non-cancer, while serum PDG, urinary NAM/Cre and Xan/Cre were downregulated
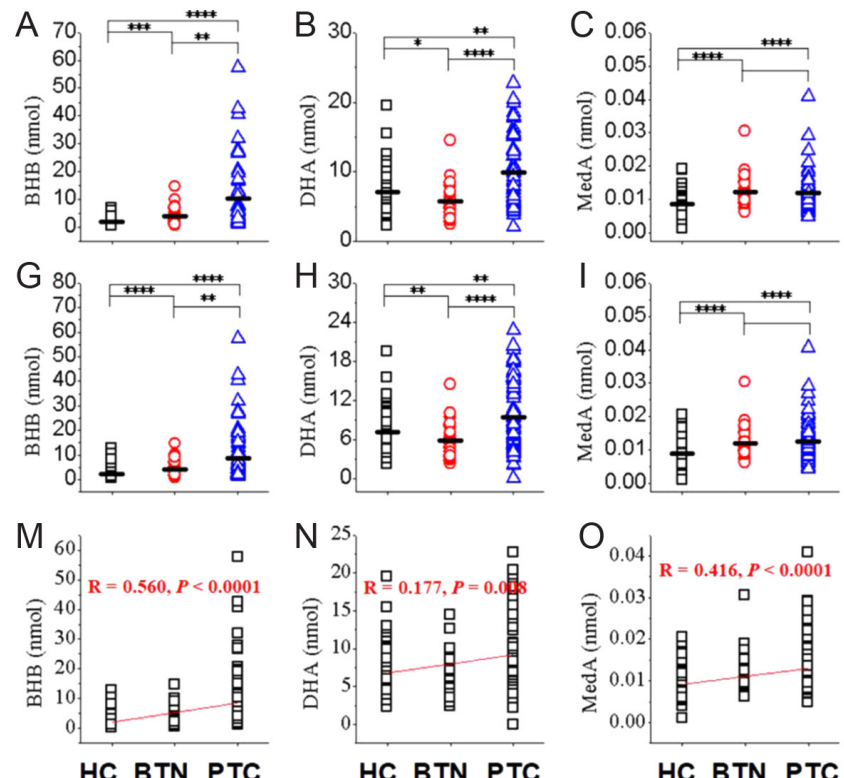
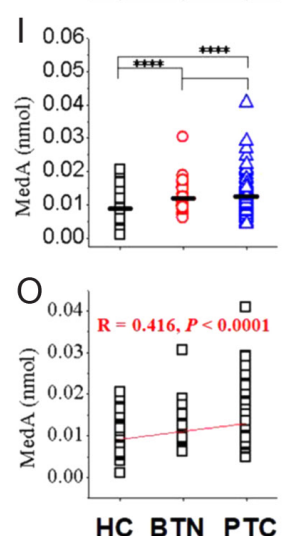
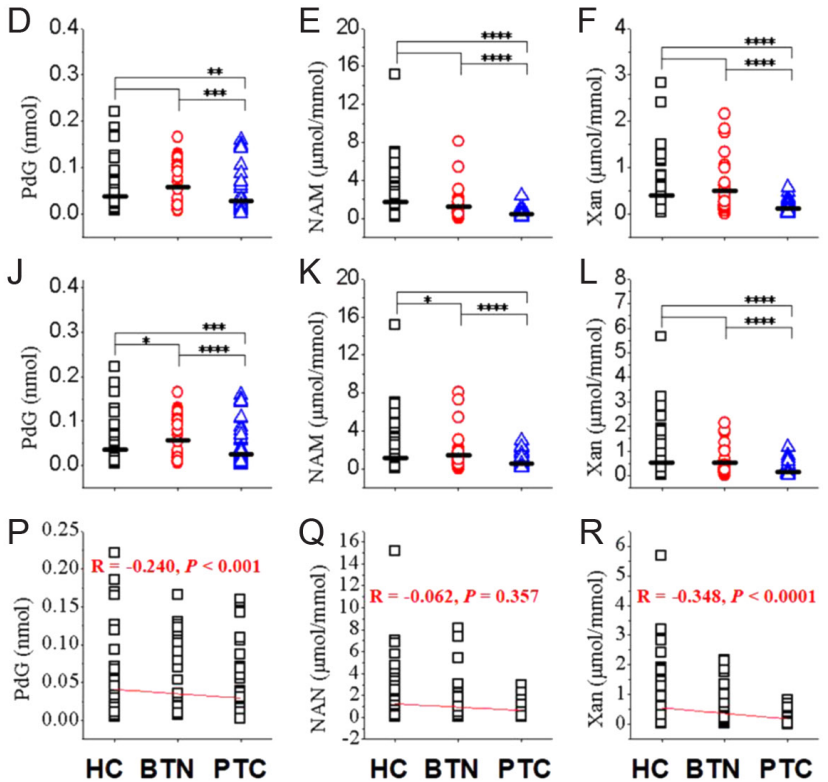

Figure 4

Differential biomarker in serum or urine of validation set samples: (A) serum BHB levels in female PTC patients, BTN patients and Healthy; (B) serum DHA levels in female PTC patients, BTN patients and Healthy; (C) serum 1-MedA levels in female PTC patients, BTN patients and Healthy; (D) serum PdG levels in female PTC patients, BTN patients and Healthy; (E) urine NAM/Cre levels in female PTC patients, BTN patients and Healthy; (F) urine xanthosine levels in female PTC patients, BTN patients and Healthy; (G) serum BHB levels in whole PTC patients, BTN patients and Healthy; (H) serum DHA levels in whole PTC patients, BTN patients and Healthy; (I) serum 1-MedA levels in whole PTC patients, BTN patients and Healthy; (J) serum PdG levels in whole PTC patients, BTN patients and Healthy; (K) urine NAM/Cre levels in whole PTC patients, BTN patients and Healthy; (L) urine xanthosine levels in whole PTC patients, BTN patients and Healthy; (I) Spearman's rank correlation of BHB with thyroid status; (J) Spearman's rank correlation of DHA with thyroid status; (K) Spearman's rank correlation of 1-MedA with thyroid status; (L) Spearman's rank correlation of PdG with thyroid status; (M) Spearman's rank correlation of NAM with thyroid status; (N) Spearman's rank correlation of Xan with thyroid status; (thyroid status: HC, BTN and PTC were defined as 1, 2 and 3, respectively.) ( $* P<0.05, \star \star p<0.01, \star \star \star * P<0.001, \star \star \star \star x<0.0001)$. A full colour version of this figure is available at https://doi.org/10.1530/ ERC-19-0344. 
in female PTC (Fig. 4A, B, C, D, E and F) and female+male PTC (Fig. 4G, H, I, J, K and L). The differential metabolites level and $P$ value in Mann-Whitney $U$ test were as follows: BHB (nmol), $P=8.85 \times 10^{-11} ;$ DHA $(\mathrm{nmol}), \quad 7.88 \times 10^{-5}$; 1-MedA (nmol), $P=0.02$; PDG (nmol), $P=1.15 \times 10^{-4}$; NAM/Cre $\quad(\mu \mathrm{mol} / \mathrm{mmol}), \quad P=1.12 \times 10^{-7} ; \quad X a n / C r e$ $(\mu \mathrm{mol} / \mathrm{mmol}), \quad P=3.09 \times 10^{-7}$. As the characters of six biomarkers' values appeared to be non-normal distributions, Box-Cox transformation method was carried out to make them close to Gaussian by Matlab. The relationships of serum/urine biomarkers levels with age or thyroid status were also analyzed in the Han Chinese volunteers aging from 24 to 74 years. No correlation was found between six biomarkers and age. Furthermore, spearman's rank correlation was carried out to explore the relationship between marker levels and thyroid status. HC, BTN, PTC were scored with 1, 2 and 3, respectively. As shown in Fig. 4M, N, O, P, Q and R, six biomarkers generally have medium correlations with thyroid status $(0.17<|R|<0.56$, $P<0.01)$ except NAM/Cre $(R=-0.06, P>0.05)$.

\section{Biomarker panel of logistic regression model}

Multivariable logistic regression model was constructed to estimate the probability of incidence for female PTC patients. Regression coefficients ( $\beta$-coefficients), odds ratio (OR, 95\% CI) and standard error (s.E.) for covariates in the logistic regression model for the probability of female PTC patients were presented in Table 2. The HosmerLemershow goodness-of-fit test showed a good calibration $\chi^{2}(8) 2.917, P=0.939$. The overall model performance evaluated using the Brier score gave a $P$ value of 0.0895 . For a perfect classifier, the AUC is close to 1.00 when the curve with $100 \%$ sensitivity and $100 \%$ specificity, whereas for a poor classifier, the ROC curve is close to the 1:1 diagonal. Biomarker panel achieved a promising prediction rate with an AUC of 0.943 (95\% CI 0.902 to 0.983) for female samples alone (Fig. 5A, B and C), demonstrating that the model established in our study represents a viable tool for PTC diagnosis. $P>0.5$ indicates the prospective incidence of the PTC, whereas $P<0.5$ indicates grade non-cancer. For female logistic regression model, the sensitivity and specificity were 81.8 and $90.0 \%$, respectively. The falsenegative rate in the real setting of one female PTC to ten female suspicious nodules was $17.9 \%$, the FPR was $16.8 \%$, and the prediction accuracy was $83.1 \%$.

For female + male PTC test set (both male and female), the risk model was rebuilt to estimate the probability of incidence for female and male PTC. Biomarker panel achieved a promising prediction rate with an AUC of 0.952 (95\% CI 0.921 to 0.983) (Fig. 5D, E and F). For female + male PTC test, the sensitivity and specificity were 81.3 and $90.7 \%$, respectively. Hosmer-Lemershow goodness-of-fit test demonstrated an excellent calibration $\chi^{2}(8) 8.508, P=0.386$. Regression coefficients, odds ratio $(95 \% \mathrm{CI})$ and s.e. for covariates in the logistic regression model for the probability of female+male PTC patients were presented in Table 2 . The overall model performance evaluated using the Brier score gave a $P$ value of 0.0857 . The false-negative rate in the real setting of 1 PTC to ten suspicious nodules was $17.7 \%$, the FPR was $15.1 \%$, and the prediction accuracy was $84.7 \%$.

Table 2 Regression coefficients ( $\beta$-coefficients), odds ratio (OR, 95\% Cl) and standard error (s.E.) for covariates in the final model for the probability of PTC.

\begin{tabular}{l}
\hline Covariates \\
\hline PTC (female) \\
BHB \\
DHA \\
MedA \\
PdG \\
NAM \\
Xan \\
Intercept \\
PTC (female + male) \\
BHB \\
DHA \\
MedA \\
PdG \\
NAM \\
Xan \\
Intercept \\
\hline
\end{tabular}

$\begin{array}{r}\hline \boldsymbol{\beta} \text {-coefficients } \\ \hline 4.010 \\ 1.283 \\ 2.545 \\ -1.042 \\ 0.286 \\ -2.246 \\ -2.322 \\ \\ 3.276 \\ 0.774 \\ 1.868 \\ -0.956 \\ 1.329 \\ -2.600 \\ -2.951 \\ \hline\end{array}$

\begin{tabular}{cc}
\hline S.E. \\
\hline 0.834 \\
0.302 \\
0.768 \\
0.371 \\
0.777 \\
0.806 \\
0.316 \\
0.556 \\
0.175 \\
0.499 \\
0.282 \\
0.392 \\
0.474 \\
2.849 \\
\hline
\end{tabular}

\begin{tabular}{c}
\hline OR $(95 \% \mathrm{Cl})$ \\
\hline $55.156(10.747$ to 283.077$)$ \\
$3.608(1.996$ to 6.521$)$ \\
$12.748(2.830$ to 57.427$)$ \\
$0.353(0.171$ to 0.729$)$ \\
$1.331(0.290$ to 6.102$)$ \\
$0.106(0.022$ to 0.513$)$ \\
- \\
$26.461(8.903$ to 78.644$)$ \\
$2.168(1.538$ to 3.057$)$ \\
$6.478(2.436$ to 17.228$)$ \\
$0.384(0.221$ to 0.668$)$ \\
$3.778(1.751$ to 8.153$)$ \\
$0.074(0.029$ to 0.188$)$ \\
-
\end{tabular}

\begin{tabular}{c}
\hline P value \\
\hline$<0.0001$ \\
$<0.0001$ \\
0.001 \\
0.005 \\
0.713 \\
0.005 \\
0.098 \\
$<0.0001$ \\
$<0.0001$ \\
0.0002 \\
0.001 \\
0.001 \\
$<0.0001$ \\
0.300 \\
\hline
\end{tabular}



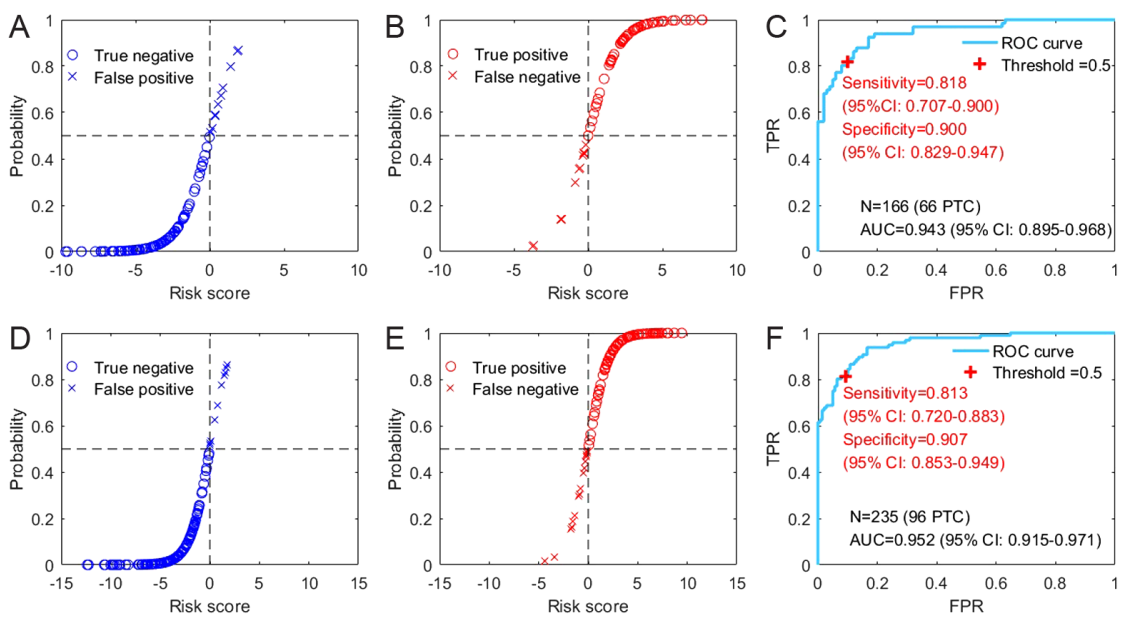

\section{Figure 5}

(A) Risk score and probability for female of non-cancer with logistic regression model; (B) Risk score and probability for female PTC with logistic regression model; (C) ROC curve plotted with false-positive rate (FPR) and true-positive rate (TPR) of logistic regression probability for female PTC classification. (D) Risk score and probability for non-cancer in female + male group with logistic regression model; (E) risk score and probability for whole PTC in logistic regression model; (F) ROC curve plotted with FPR and TPR of logistic regression probability for female + male PTC classification. A full colour version of this figure is available at https://doi.org/10.1530/ ERC-19-0344.

\section{Double-blind study}

As the sample information and the results of the clinical diagnosis were unknown to the laboratory technician, the risk model based on female + male PTC test set was used to predict the blind samples. As shown in Table 3, the misclassification rate, calculated as undiagnosed divided by the total sample size, was $6.5 \%$. The true-positive and true-negative values were 100 and $91.7 \%$, respectively. Double-blind study revealed that the logistic regression model built in this research performed well, and the method could be used as an auxiliary tool for clinical diagnosis.

\section{Discussion}

Veyrat-Durebex et al. conveyed a metabolomic study on metabolomics signatures with a subset of RET variants according to their oncogenic risk level and demonstrated the efficacy of metabolomics in testing RET mutations' pathogenicity (Veyrat-Durebex et al. 2019). A serumplasma-matched untargeted metabolomics study was conducted to reveal the significant metabolic pattern differences of BTN and PTC, and no significant difference was found in circulating metabolic signatures between

Table 3 Double-blind study result based on logistic regression model placed on a risk score for real setting of PTC to suspicious nodules.

\begin{tabular}{|c|c|c|c|c|}
\hline \multirow[b]{2}{*}{ Outcome } & \multicolumn{3}{|c|}{ The result } & \\
\hline & True & False & Total & \\
\hline PTC & 7 & 0 & 7 & True positive = 7/7 (100\%) \\
\hline BTN & 22 & 2 & 24 & True negative $=22 / 24(91.7 \%)$ \\
\hline Total & 29 & 2 & 31 & \\
\hline
\end{tabular}

BTN and PTC (Huang et al. 2019). For differential diagnosis of HC versus BTN and PTC, a panel of six metabolic markers, namely myo-inositol, N-phenylacetyl-Lglutamine, proline betaine, L-glutamic acid, LysoPC (18:0) and LysoPC (18:1) provided AUC of $97.68 \%$ in discovery phase and predictive accuracies of $84.78-98.18 \%$ (Huang et al. 2019). However, there are still some deficiencies, such as lacking validation or limited validation objects of early diagnosis of PTC biomarkers from BTN and HC.

In this article, differential metabolites were screened from non-targeted metabolomics with a discovery sample set, and then validated by targeted metabolomics and corrected with creatinine in another validation set. Thirty-six potential differential metabolites identified by high-throughput screening were included in serum or urine. Six candidate markers were selected for further testing as they represented different metabolic pathways associated with cancer. 1-MedA, one of the modified nucleosides involved in the purine metabolism, was found downregulated in serum, and the levels were disordered in urine of patients with malignant tumors (Ishiwata et al. 1995). Ketone bodies played both metabolic and epigenetic roles in cancer, while $\beta$-hydroxybutyrate (BHB) was involved in the synthesis and degradation of ketone bodies. Some studies proposed that ketone bodies could inhibit histone deacetylases from achieving anticancer effect (Seyfried et al. 2003, Poff et al. 2014, Shukla et al. 2014), while other studies noted faster tumor growth (Bonuccelli et al. 2010, Whitakermenezes et al. 2011, Martinezoutschoorn et al. 2012, Rodrigues et al. 2017, Wojtowicz et al. 2017). Rodrigues found that BHB administration accelerated the rate of tumor growth rather than inhibited it (Rodrigues et al. 2017). Hence, upregulation of BHB seems to be an important element related to PTC. DHA is a member of 
long-chain fatty acids, which can be obtained from seafood consumption. Previous study proposed that intake of salt water fish, fresh water fish, cruciferous vegetables, fruit, meat, and grains were not related with the risk of thyroid cancer (Liu \& Lin 2014). However, DHA may also be synthesized endogenously from linolenic acid. Serum DHA concentration was found significantly upregulated in PTC in our study, which might be affected by abnormal citric acid metabolism. PdG was a natural metabolite generated by UDP glucuonyltransferase and pregnanediol, whose upregulation in urine was known as a biomarker to confirm ovulation (Souza et al. 1998; Ecochard et al. 2013). Previous studies found that the urine estrone-glucuronide/PdG ratio was significantly increased in the women with breast cancer, in spite of normal pregnanediol-3-glucuronide excretion (Magini et al. 1990). NaMN was an intermediate in the metabolism of nicotinate and nicotinamide, and its acetylation was based on the synthetic pathway of deamination from NAD (Wang et al. 2017b). Inhibition of nicotinamide phosphate transferase has become a therapeutic strategy in cancer (Santidrian et al. 2013, Sampath et al. 2015). Xan, a purine nucleoside, is an intermediate in purine metabolism (Stoychev et al. 2002, López-Cruz et al. 2014), while it is an important metabolic process of cell proliferation (Zhou et al. 2017). Notably, untargeted results revealed that serum $\mathrm{BHB}$, DHA and 1-MedA were dramatically upregulated in PTC compared with noncancer group, and serum PdG, urinary NAM and Xan were significantly downregulated in PTCs. The six metabolic biomarkers were upregulated/downregulated by targeting metabolomics.

We firstly studied the relationship between the six metabolic markers and age as well as thyroid status. For the population aging from 20 to 74 years: there was no correlation between six biomarkers and age. Although presented results of spearman's rank correlation indicated that six markers except NAM were significantly related to thyroid status, a larger sample size is needed for further validation and PTC stage classification developments. Staging and prognosis require independent sample analysis, while the number of patients with advanced disease and recurrence is too small to draw a final conclusion.

For the application perspective, our research aims to develop a predictive assay that can help early diagnosis of PTC. Since a single metabolite cannot be a universal marker, six metabolic biomarkers were combined as a metabolic biomarker panel and applied with logistic regression model to evaluate the potential diagnostic performance, which provided a satisfactory prediction accuracy of
$84.7 \%$ (threshold at probability of 0.5 ) through training and validation for identifying PTC from $\mathrm{HC}$ as well as BTN. The result is comparable to the predicted value of available clinical markers reported so far: mutation testing panel (BRAF, RAS mutations, and PAX8-PPARG, RET/PTC1,3) (81-94\%) (Nikiforov et al. 2011), HMGA gene (91.2\%) (Vandergrift et al. 2018), Kronus/RSR TgAb (89\%), Roche Elecsys TgAb (85\%) (Spencer et al. 2014), and metabolic tumor volume ( $\triangle \mathrm{MTV} \%, 87.2 \%)$ and total lesion glycolysis ( $\triangle \mathrm{TLG} \%, 89.5 \%$ ) in PET/CT (Shi et al. 2018), and F-18 fluorodeoxyglucose (F-18 FDG, 0.84) in PET/CT (Kim et al. 2018). Specifically, low-dose CT screening is expensive, characterized by high sensitivity but only 68.6\% specificity (Shi et al. 2018). Although diagnostic value can be improved combining with measurable PTC markers, the sensitivity and specificity of these markers are limited, which cannot be widely used in clinical practice (Saleh et al. 2010, Alexander et al. 2012, Ohori et al. 2013, Xing et al. 2013). The results presented in this article would help to compare existing and new developments in the field objectively. The additional validation should include a larger scale of subjects, and these subjects' age and gender should be carefully matched, including individuals with various benign thyroid disease and high-risk groups. Advanced methods will be built and tested with the provided data for further study.

\section{Conclusions}

In summary, serum BHB, DHA, 1-MedA, PdG, and urinary NAM/Cre and Xan/Cre were screened to be differential metabolites by non-targeted metabolomics with a discovery sample set, and then validated to be potential markers by targeted metabolomics in another validation set. The six biomarkers had significant correlations with thyroid status except NAM. For the application in clinical analysis, the PTC prediction accuracy was assayed with a novel diagnosis strategy based on logistic regression model with biomarker panel. The risk model was proved to be highly sensitive, specific, and well matched after bootstrapping and double-blind validation. In addition, the model also might be developed for auxiliary diagnosis of PTC patients with further validation.

Supplementary data

This is linked to the online version of the paper at https://doi.org/10.1530/ ERC-19-0344. (c) 2019 Society for Endocrinology Published by Bioscientifica Ltd. Printed in Great Britain 


\section{Declaration of interest}

The authors declare that there is no conflict of interest that could be perceived as prejudicing the impartiality of the research reported.

\section{Funding}

This work was financially supported by National Natural Science Foundation of China (21277174).

\section{Ethics approval and consent to participate}

This study was approved by the ethics committee of School of Basic Medical Sciences, Zhengzhou University (Approval number 18).

\section{Acknowledgments}

The authors gratefully thank the Ethics Committee of School of Basic Medical Sciences, Zhengzhou University, the Tumor Hospital affiliated to Zhengzhou University and Zhengzhou Central Hospital affiliated to Zhengzhou University.

\section{References}

Alexander EK, Kennedy GC, Baloch ZW, Cibas ES, Darya C, James D, Lyssa F, Kloos RT, Livolsi VA \& Mandel SJ 2012 Preoperative diagnosis of benign thyroid nodules with indeterminate cytology. New England Journal of Medicine 367 705-715. (https://doi. org/10.1056/NEJMoa1203208)

Bonuccelli G, Tsirigos A, Whitaker-Menezes D, Pavlides S, Pestell RG, Chiavarina B, Frank PG, Flomenberg N, Howell A, MartinezOutschoorn UE, et al. 2010 Ketones and lactate fuel tumor growth and metastasis: evidence that epithelial cancer cells use oxidative mitochondrial metabolism. Cell Cycle 9 3506-3514. (https://doi. org/10.4161/cc.9.17.12731)

Bustamante E, Morris HP \& Pedersen PL 1981 Energy metabolism of tumor cells. Requirement for a form of hexokinase with a propensity for mitochondrial binding. Journal of Biological Chemistry 256 8699-8704.

Ceglarek U, Leichtle A, Brügel M, Kortz L, Brauer R, Bresler K, Thiery J \& Fiedler GM 2009 Challenges and developments in tandem mass spectrometry based clinical metabolomics. Molecular \& Cellular Endocrinology 301 266-271. (https://doi.org/10.1016/j. mce.2008.10.013)

Coperchini F, Croce L, Marinò M, Chiovato L \& Rotondi M 2019 Role of chemokine receptors in thyroid cancer and immunotherapy. Endocrine-Related Cancer 26 465-478. (https://doi.org/10.1530/ERC19-0163)

Dunn WB, Broadhurst D, Begley P, Zelena E, Francismcintyre S, Anderson N, Brown M, Knowles JD, Halsall A, Haselden JN, et al. 2011 Procedures for large-scale metabolic profiling of serum and plasma using gas chromatography and liquid chromatography coupled to mass spectrometry. Nature Protocols 6 1060-1083. (https:// doi.org/10.1038/nprot.2011.335)

Ecochard R, Leiva R, Bouchard T, Boehringer H, Direito A, Mariani A \& Fehring R 2013 Use of urinary pregnanediol 3-glucuronide to confirm ovulation. Steroids $\mathbf{7 8} 1035-1040$. (https://doi.org/10.1016/j. steroids.2013.06.006)

Goodwin ML, Gladden LB, Nijsten MWN \& Jones KB 2014 Lactate and cancer: revisiting the Warburg effect in an era of lactate shuttling. Frontiers in Nutrition 1 27. (https://doi.org/10.3389/fnut.2014.00027)
Haugen BR, Alexander EK, Bible KC, Doherty GM, Mandel SJ, Nikiforov YE, Pacini F, Randolph GW, Sawka AM \& Schlumberger 20162015 American Thyroid Association management guidelines for adult patients with thyroid nodules and differentiated thyroid cancer: the American Thyroid Association Guidelines Task Force on Thyroid Nodules and Differentiated Thyroid Cancer. Thyroid 26 1-133. (https://doi.org/10.1089/thy.2015.0020)

Huang FQ, Li J, Jiang L, Wang FX, Alolga RN, Wang MJ, Min WJ, Ma G, Zhao YJ, Wang SL, Yu Y, Chen X, Zhu D, Zhu J \& Wang G 2019 Serum-plasma matched metabolomics for comprehensive characterization of benign thyroid nodule and papillary thyroid carcinoma. International Journal of Cancer 4 868-876. (https://doi. org/10.1002/ijc.31925)

Ishiwata S, Itoh K, Yamaguchi T, Ishida N \& Mizugaki M 1995 Comparison of serum and urinary levels of modified nucleoside, 1-methyladenosine, in cancer patients using a monoclonal antibodybased inhibition ELISA. Tohoku Journal of Experimental Medicine 176 61-68. (https://doi.org/10.1620/tjem.176.61)

Jones GRD, Haeckel R, Loh TP, Sikaris K, Streichert T, Katayev A, Barth JH, Ozarda Y \& IFCC Committee on Reference Intervals and Decision Limits 2018 Indirect methods for reference interval determination - review and recommendations. Clinical Chemistry \& Laboratory Medicine 57 20-29. (https://doi.org/10.1515/cclm-20180073)

Kim SJ, Lee SW, Pak K \& Shim SR 2018 Diagnostic performance of PET in thyroid cancer with elevated anti-Tg ab. Endocrine-Related Cancer 25 643-652. (https://doi.org/10.1530/ERC-17-0341)

La Vecchia C, Malvezzi M, Bosetti C, Garavello W, Bertuccio P, Levi F \& Negri E 2015 Thyroid cancer mortality and incidence: a global overview. International Journal of Cancer 136 2187-2195. (https://doi. org/10.1002/ijc.29251)

Levey AS, Perrone RD \& Madias NE 1988 Serum creatinine and renal function. Annual Review of Medicine 39 465-490. (https://doi. org/10.1146/annurev.me.39.020188.002341)

Lin JS, Bowles EJA, Williams SB \& Morrison CC 2017 Screening for thyroid cancer: updated evidence report and systematic review for the US Preventive Services Task Force. JAMA 317 1888-1903. (https://doi.org/10.1001/jama.2017.0562)

Liu ZT \& Lin AH 2014 Dietary factors and thyroid cancer risk: a metaanalysis of observational studies. Nutrition \& Cancer 66 1165-1178. (https://doi.org/10.1080/01635581.2014.951734)

Lloyd RV, Osamura RY, Kloppel G \& Rosai J 2017 WHO Classification of Tumours of Endocrine Organs, 4th ed. Lyon, France: IARC.

Locasale JW 2013 Serine, glycine and one-carbon units: cancer metabolism in full circle. Nature Reviews. Cancer 13 572-583. (https://doi.org/10.1038/nrc3557)

López-Cruz RI, Pérez-Milicua MB, Crocker DE, Gaxiola-Robles R, BernalVertiz JA, de la Rosa A, Vázquez-Medina JP \& Zenteno-Savín T 2014 Purine nucleoside phosphorylase and xanthine oxidase activities in erythrocytes and plasma from marine, semiaquatic and terrestrial mammals. Comparative Biochemistry and Physiology Part A 171 31-35. (https://doi.org/10.1016/j.cbpa.2014.02.007)

Magini A, Pazzagli M, Salerno R, Simonis M, Mustacchi G \& Serio M 1990 Ovarian function in premenopausal women affected by breast cancer: the measurement of glucuronoconjugate metabolites of 17 beta-estradiol and progesterone throughout one entire menstrual cycle. Journal of Steroid Biochemistry 36 523-526. (https://doi. org/10.1016/0022-4731(90)90168-r)

Martinezoutschoorn UE, Lin Z, Whitakermenezes D, Howell A, Sotgia F \& Lisanti MP 2012 Ketone body utilization drives tumor growth and metastasis. Cell Cycle 11 3964-3971. (https://doi.org/10.4161/ cc.22137)

Nicholson JK \& Lindon JC 2008 Systems biology: metabonomics. Nature 455 1054-1056. (https://doi.org/10.1038/4551054a)

Nikiforov YE, Ohori NP, Hodak SP, Carty SE, LeBeau SO, Ferris RL, Yip L, Seethala RR, Tublin ME, Stang MT, et al. 2011 Impact of mutational (c) 2019 Society for Endocrinology Published by Bioscientifica Ltd. Printed in Great Britain 
testing on the diagnosis and management of patients with cytologically indeterminate thyroid nodules: a prospective analysis of 1056 FNA samples. Journal of Clinical Endocrinology \& Metabolism 96 3390-3397. (https://doi.org/10.1210/jc.2011-1469)

Ohori NP, Singhal R, Nikiforova MN, Yip L, Schoedel KE, Coyne C, Mccoy KL, Lebeau SO, Hodak SP, Carty SE, et al. 2013 BRAF mutation detection in indeterminate thyroid cytology specimens: underlying cytologic, molecular, and pathologic characteristics of papillary thyroid carcinoma. Cancer Cytopathology 121 197-205. (https://doi.org/10.1002/cncy.21229)

Pan L, Aguilar HA, Wang L, Iliuk A \& Tao WA 2016 Three-dimensionally functionalized reverse phase glycoprotein array for cancer biomarker discovery and validation. Journal of the American Chemical Society 138 15311-15314. (https://doi.org/10.1021/jacs.6b10239)

Poff AM, Ari C, Arnold P, Seyfried TN \& D'Agostino DP 2014 Ketone supplementation decreases tumor cell viability and prolongs survival of mice with metastatic cancer. International Journal of Cancer 135 1711-1720. (https://doi.org/10.1002/ijc.28809)

Ravetto C, Colombo L \& Dottorini ME 2000 Usefulness of fine-needle aspiration in the diagnosis of thyroid carcinoma: a retrospective study in 37,895 patients. Cancer $90357-363$. (https://doi. org/10.1002/1097-0142(20001225)90:6<357::AID-CNCR6>3.0.CO;2-4)

Rodrigues LM, Uribe-Lewis S, Madhu B, Honess DJ, Stubbs M \& Griffiths JR 2017 The action of $\beta$-hydroxybutyrate on the growth, metabolism and global histone $\mathrm{H} 3$ acetylation of spontaneous mouse mammary tumours: evidence of a $\beta$-hydroxybutyrate paradox. Cancer \& Metabolism 5 4. (https://doi.org/10.1186/s40170017-0166-z)

Ryoo I, Kwon H, Kim SC, Jung SC, Yeom JA, Shin HS, Cho HR, Yun TJ, Choi SH, Sohn CH, et al. 2016 Metabolomic analysis of percutaneous fine-needle aspiration specimens of thyroid nodules: potential application for the preoperative diagnosis of thyroid cancer. Scientific Reports 6 30075. (https://doi.org/10.1038/srep30075)

Saleh HA, Jin B, Barnwell J \& Alzohaili O 2010 Utility of immunohistochemical markers in differentiating benign from malignant follicular-derived thyroid nodules. Diagnostic Pathology 5 9. (https://doi.org/10.1186/1746-1596-5-9)

Sampath D, Zabka TS, Misner DL, O'Brien T \& Dragovich PS 2015 Inhibition of nicotinamide phosphoribosyltransferase (NAMPT) as a therapeutic strategy in cancer. Pharmacology \& Therapeutics 151 1631. (https://doi.org/10.1016/j.pharmthera.2015.02.004)

Santidrian AF, Matsunoyagi A, Ritland M, Seo BB, Leboeuf SE, Gay LJ, Yagi T \& Feldinghabermann B 2013 Mitochondrial complex I activity and NAD+/NADH balance regulate breast cancer progression. Journal of Clinical Investigation 123 1068-1081. (https://doi. org/10.1172/JCI64264)

Seyfried TN, Sanderson TM, El-Abbadi MM, Mcgowan R \& Mukherjee P 2003 Role of glucose and ketone bodies in the metabolic control of experimental brain cancer. British Journal of Cancer 89 1375-1382. (https://doi.org/10.1038/sj.bjc.6601269)

Shi H, Yuan Z, Yuan Z, Yang C, Zhang J, Shou Y, Zhang W, Ping Z, Gao X \& Liu S 2018 Diagnostic value of volume-based fluorine-18fluorodeoxyglucose PET/CT parameters for characterizing thyroid incidentaloma. Korean Journal of Radiology 19 342-351. (https://doi. org/10.3348/kjr.2018.19.2.342)

Shukla SK, Gebregiworgis T, Purohit V, Chaika NV, Gunda V, Radhakrishnan P, Mehla K, Pipinos II, Powers R \& Yu F 2014 Metabolic reprogramming induced by ketone bodies diminishes pancreatic cancer cachexia. Cancer \& Metabolism 2 18. (https://doi. org/10.1186/2049-3002-2-18)

Souza MJD, Miller BE, Loucks AB, Luciano AA, Pescatello LS, Campbell CG \& Lasley BL 1998 High frequency of luteal phase deficiency and anovulation in recreational women runners: blunted elevation in follicle-stimulating hormone observed during lutealfollicular transition. Journal of Clinical Endocrinology \& Metabolism $\mathbf{8 3}$ 4220-4232. (https://doi.org/10.1210/jcem.83.12.5334)
Spencer C, Petrovic I, Fatemi S \& LoPresti J 2014 Serum thyroglobulin (Tg) monitoring of patients with differentiated thyroid cancer using sensitive (second-generation) immunometric assays can be disrupted by false-negative and false-positive serum thyroglobulin autoantibody misclassifications. Journal of Clinical Endocrinology \& Metabolism 99 4589-4599. (https://doi.org/10.1210/jc.2014-1203)

Stoychev G, Kierdaszuk B \& Shugar D 2002 Xanthosine and xanthine. Substrate properties with purine nucleoside phosphorylases, and relevance to other enzyme systems. European Journal of Biochemistry 269 4048-4057. (https://doi.org/10.1046/j.1432-1033.2002.03097.x)

Stoychev G, Kierdaszuk B \& Shugar D 2010 Xanthosine and xanthine. Substrate properties with purine nucleoside phosphorylases, and relevance to other enzyme systems. FEBS Journal 269 4048-4057.

Tian Y, Nie X, Xu S, Li Y, Huang T, Tang H \& Wang Y 2015 Integrative metabonomics as potential method for diagnosis of thyroid malignancy. Scientific Reports 5 14869. (https://doi.org/10.1038/ srep14869)

van Velsen EFS, Stegenga MT, van Kemenade FJ, Kam BLR, van Ginhoven TM, Visser WE \& Peeters RP 2018 Comparing the prognostic value of the eighth edition of the American Joint Committee on cancer/tumor node metastasis staging system between papillary and follicular thyroid cancer. Thyroid 28 976-981. (https:// doi.org/10.1089/thy.2018.0066)

Vandergrift LA, Decelle EA, Kurth J, Wu S, Fuss TL, DeFeo EM, Halpern EF, Taupitz M, McDougal WS, Olumi AF, et al. 2018 Metabolomic prediction of human prostate cancer aggressiveness: magnetic resonance spectroscopy of histologically benign tissue. Scientific Reports 8 4997. (https://doi.org/10.1038/s41598-018-23177-w)

Veyrat-Durebex C, Bouzamondo N, Le Mao M, Chao de la Barca JM, Bris C, Dieu X, Simard G, Gadras C \& Tessier L 2019 Metabolomics signatures of a subset of RET variants according to their oncogenic risk level. Endocrine-Related Cancer 3 379-389. (https://doi. org/10.1530/ERC-18-0314)

Wang CCC, Friedman L, Kennedy GC, Wang H, Kebebew E, Steward DL, Zeiger MA, Westra WH, Wang Y, Khanafshar E, et al. 2011 A large multicenter correlation study of thyroid nodule cytopathology and histopathology. Thyroid 21 243-251. (https://doi.org/10.1089/ thy.2010.0243)

Wang Y, Pei H, Jia Y, Liu J, Li Z, Ai K, Lu Z \& Lu L 2017a Synergistic tailoring of electrostatic and hydrophobic interactions for rapid and specific recognition of lysophosphatidic acid, an early-stage ovarian cancer biomarker. Journal of the American Chemical Society 139 11616-11621. (https://doi.org/10.1021/jacs.7b06885)

Wang X, Zhou YJ, Wang L, Liu W, Liu Y, Peng C \& Zhao ZK 2017b Engineering Escherichia coli nicotinic acid mononucleotide adenylyltransferase for fully active amidated NAD biosynthesis. Applied \& Environmental Microbiology 83 00692-00617. (https://doi. org/10.1128/AEM.00692-17)

Whitakermenezes D, Martinezoutschoorn UE, Lin Z, Ertel A, Flomenberg N, Witkiewicz AK, Birbe RC, Howell A, Pavlides S, Gandara R, et al. 2011 Evidence for a stromal-epithelial "lactate shuttle" in human tumors: MCT4 is a marker of oxidative stress in cancer-associated fibroblasts. Cell Cycle 10 1772-1783. (https://doi. org/10.4161/cc.10.11.15659)

Wise DR \& Thompson CB 2010 Glutamine addiction: a new therapeutic target in cancer. Trends in Biochemical Sciences 35 427-433. (https:// doi.org/10.1016/j.tibs.2010.05.003)

Wojakowska A, Chekan M, Widlak P \& Pietrowska M 2015a Application of metabolomics in thyroid cancer research. International Journal of Endocrinology 2015 258763. (https://doi.org/10.1155/2015/258763)

Wojakowska A, Chekan M, Marczak Ł, Polanski K, Lange D, Pietrowska M \& Widlak P 2015b Detection of metabolites discriminating subtypes of thyroid cancer: molecular profiling of FFPE samples using the GC/MS approach. Molecular \& Cellular Endocrinology 417 149-157. (https://doi.org/10.1016/j. mce.2015.09.021) (c) 2019 Society for Endocrinology Published by Bioscientifica Ltd. Printed in Great Britain 
Wojtowicz W, Zabek A, Deja S, Dawiskiba T, Pawelka D, Glod M, Balcerzak W \& Mlynarz P 2017 Serum and urine 1H NMR-based metabolomics in the diagnosis of selected thyroid diseases. Scientific Reports 7 9108. (https://doi.org/10.1038/s41598-017-09203-3)

Xing M, Haugen BR \& Schlumberger M 2013 Progress in molecular-based management of differentiated thyroid cancer. Lancet 381 1058-1069. (https://doi.org/10.1016/S01406736(13)60109-9)

Xu Y, Zheng X, Qiu Y, Jia W, Wang J \& Yin S 2015 Distinct metabolomic profiles of papillary thyroid carcinoma and benign thyroid adenoma. Journal of Proteome Research 14 3315-3321. (https://doi.org/10.1021/ acs.jproteome.5b00351)
Yao Z, Yin P, Su D, Peng Z, Zhou L, Ma L, Guo W, Ma L, Xu G, Shi J, et al. 2011 Serum metabolic profiling and features of papillary thyroid carcinoma and nodular goiter. Molecular Biosystems 72608 2614. (https://doi.org/10.1039/c1mb05029j)

Zafón Llopis C, Gil J, Pérez-González B \& Jordà M 2019 DNA methylation in thyroid cancer. Endocrine-Related Cancer 26 415-439. (https://doi.org/10.1530/ERC-19-0093)

Zhou Y, Song R, Ma C, Zhou L, Liu X, Yin P, Zhang Z, Sun Y, Xu C, Lu X, et al. 2017 Discovery and validation of potential urinary biomarkers for bladder cancer diagnosis using a pseudotargeted GC-MS metabolomics method. Oncotarget 8 20719-20728. (https:// doi.org/10.18632/oncotarget.14988)

Received in final form 27 September 2019

Accepted 30 September 2019

Accepted Preprint published online 1 October 2019 (c) 2019 Society for Endocrinology Published by Bioscientifica Ltd. Printed in Great Britain 